\section{Estrategia mundial de la OMS para contener la resistencia a los antimicrobianos $^{1}$}

Palabras clave: antimicrobianos, OMS, resistencia.

Basado en el documento WHO global strategy for containment of antimicrobial resistance. Geneva: World Health Organization; 2001 (WHO/CDS/CSR/DRS/2001.2), disponible en http://www.who. int/emc/amr_interventions.htm
Las infecciones respiratorias agudas, las enfermedades diarreicas, el sarampión, el sida, el paludismo y la tuberculosis son responsables de más del 85\% de la mortalidad por causas infecciosas en el mundo. La resistencia de los respectivos agentes patógenos a los medicamentos de primera línea va desde cero hasta casi $100 \%$ y, en algunos casos, la resistencia a los fármacos de segunda y tercera línea compromete seriamente el resultado del tratamiento.

La resistencia no es un fenómeno nuevo, pero el desarrollo de nuevas familias de antimicrobianos en los años cincuenta y sesenta y las modificaciones de esas moléculas en los años setenta y ochenta crearon una falsa sensación de seguridad y la creencia de que siempre podríamos adelantarnos a los agentes patógenos. Al comenzar el nuevo siglo, estamos pagando muy caro esa complacencia. El desarrollo de nuevos antimicrobianos se está estancando y hay pocos incentivos para elaborar otros nuevos que permitan combatir el problema mundial de la resistencia.

La resistencia cuesta dinero y vidas humanas, pone en peligro la eficacia de los programas de atención de la salud y podría llegar a constituir una amenaza para la estabilidad mundial y la seguridad de los países. Su causa principal es el uso de los antimicrobianos y, más concretamente, la combinación del uso excesivo que se hace en muchas partes del mundo, del uso incorrecto por falta de acceso a tratamientos apropiados y de la infrautilización debido a la falta de recursos financieros para terminar los tratamientos.

La resolución WHA51.171 de la Asamblea Mundial de la Salud de 1998 instaba a los Estados Miembros a que adoptaran medidas encaminadas a promover el uso apropiado de los antimicrobianos; a prohibir su dispensación sin la prescripción de un profesional calificado; a mejorar las prácticas para prevenir la propagación de las infecciones y la consiguiente propagación de patógenos resistentes; a reforzar la legislación para impedir la fabricación, venta y distribución de antimicrobianos falsificados y la venta de antibióticos en el mercado paralelo; a reducir el uso de antimicrobianos en la cría de animales destinados al consumo humano, y a desarrollar sistemas sostenibles para detectar los patógenos resistentes y monitorear las cantidades y modalidades de uso de los antimicrobianos y los efectos de las medidas de control. Desde entonces, muchos países han expresado una creciente preocupación 
por el problema y algunos han elaborado planes nacionales de acción para hacerle frente. A pesar de que hay abundante bibliografía sobre el tema, se ha publicado poco sobre los costos reales de la resistencia y la eficacia de las intervenciones.

La Estrategia mundial de la OMS para contener la resistencia a los antimicrobianos proporciona un marco de intervenciones encaminadas a reducir la aparición y propagación de microorganismos resistentes mediante las siguientes medidas:

- reducción de la morbilidad y propagación de las infecciones;

- mejora del acceso a los antimicrobianos apropiados;

- mejora del uso de los antimicrobianos;

- fortalecimiento de los sistemas de salud y de su capacidad de vigilancia;

- cumplimiento de los reglamentos y de la legislación;

- fomento del desarrollo de nuevos medicamentos $\mathrm{y}$ vacunas.

Aunque se centra en los antibacterianos, la estrategia aborda la resistencia a los antimicrobianos en general y destaca los aspectos principales de su contención y la necesidad de nuevas investigaciones para colmar la falta de conocimientos. La estrategia está orientada hacia las personas y prevé intervenciones dirigidas a los grupos interesados en el problema que deben participar en su solución, es decir, médicos, farmacéuticos, veterinarios, consumidores, instancias normativas de hospitales y sectores de la salud pública y la agricultura, asociaciones profesionales e industria farmacéutica. Gran parte de la responsabilidad de su aplicación recaerá en cada uno de los países y los gobiernos tendrán que desempeñar un papel decisivo en el suministro de bienes públicos, como la información, en la vigilancia, en el análisis del costo-efectividad y en la coordinación multisectorial.

Dada la naturaleza compleja del problema, la estrategia comprende necesariamente un gran número de recomendaciones para diferentes intervenciones (véanse los recuadros) y la asignación de prioridades deberá ajustarse a la realidad de cada país. Para contribuir a este proceso, se ha definido un enfoque para aplicar la estrategia y se han establecido indicadores para monitorear su aplicación y resultados. El reconocimiento del problema y la creación de grupos de estudio intersectoriales nacionales eficaces son indispensables para el éxito de la aplicación y monitoreo de las intervenciones. La cooperación interdisciplinaria internacional también será esencial.

Para contener la resistencia será fundamental mejorar el uso de los antimicrobianos y para ello será preciso mejorar el acceso y modificar comportamientos, cambios que llevan tiempo. Asimismo, será necesario un fortalecimiento significativo de los sistemas de salud en muchos países, y los costos de la aplicación no serán despreciables. Sin embargo, esos costos se deben comparar con el ahorro que se obtendrá en el futuro gracias a la contención de la resistencia generalizada a los antimicrobianos.

\section{PACIENTES Y COMUNIDAD EN GENERAL}

La resistencia es un problema complejo provocado por numerosos factores interrelacionados, entre los que destaca el uso de los antimicrobianos, y especialmente su uso indebido. A su vez, el uso de antimicrobianos está influido por la interrelación entre conocimientos, expectativas e interacciones entre el que prescribe y el paciente, incentivos económicos, características del sistema sanitario y entorno legislativo. En vista de la complejidad, hacen falta intervenciones coordinadas dirigidas, a la vez, a los prestadores de atención y a los pacientes; también habrá que alterar algunas características importantes del entorno en que se produce la interacción. Si bien las intervenciones dirigidas a los prestadores de atención y a los pacientes se exponen por separado, para que resulten más claras deberán realizarse de un modo integrado. Es muy probable que las intervenciones den resultados satisfactorios, si se entienden los siguientes factores de cada uno de los entornos sanitarios:

- qué enfermedades infecciosas y qué problemas de resistencia son importantes;

- qué antimicrobianos se utilizan y quién los usa;

- qué factores determinan las modalidades de uso de los antimicrobianos;

- qué ventajas y costos relativos se derivan de las modificaciones del uso;

- qué problemas plantea el cambio de uso.

Los factores relacionados con el paciente se encuentran entre los principales condicionantes del uso indebido de antimicrobianos. Entre ellos destacan los conceptos equivocados, la automedicación, la publicidad y promoción de los medicamentos y el mal cumplimiento de los tratamientos.

\section{MÉDICOS Y FARMACÉUTICOS}

Por otra parte, también es fundamental mejorar las prácticas de prescripción y dispensación de fármacos. Sin embargo, esto no resulta fácil, dado el gran número de factores que influyen sobre dichas prácticas; además, la importancia relativa de cada 


\section{Pacientes y comunidad en general}

\section{Educación}

1.1 Educar a los pacientes y a la comunidad en general sobre el uso adecuado de los antimicrobianos.

1.2 Educar a los pacientes sobre la importancia de las medidas de prevención de las infecciones, como la inmunización y la lucha contra los vectores.

1.3 Enseñar a los pacientes medidas sencillas que pueden reducir la transmisión de las infeccio- nes, como el lavado de las manos, la higiene alimentaria, etc.

1.4 Alentar un comportamiento adecuado e informado de búsqueda de asistencia sanitaria.

1.5 Enseñar a los pacientes que existen otras opciones para aliviar los síntomas y desalentar el inicio del tratamiento por iniciativa propia, salvo en determinadas circunstancias.

\section{Médicos y farmacéuticos}

\section{Educación}

2.1 Educar a toda persona que prescriba o dispense antimicrobianos sobre la importancia de usar adecuadamente estos fármacos y de contener la resistencia.

2.2 Educar a todos los grupos de personas que prescriben antimicrobianos sobre la prevención de las enfermedades y la lucha contra las infecciones.

2.3 Promover programas educativos sobre el diagnóstico y tratamiento de las infecciones comunes destinados a todos los profesionales de la salud, veterinarios y personal que prescribe y dispensa antimicrobianos.

2.4 Alentar a todo el que prescribe o dispensa antimicrobianos a que enseñe a los pacientes su uso apropiado y la importancia de cumplir los tratamientos prescritos.

2.5 Educar a todos los grupos de profesionales que prescriben o dispensan antimicrobianos sobre los factores que pueden influir en sus hábitos de prescripción, como los incentivos económicos, las actividades de promoción y los estímulos de la industria farmacéutica.
Tratamiento, directrices y formularios

2.6 Mejorar el uso de los antimicrobianos mediante la supervisión y el fomento de buenas prácticas clínicas, y especialmente de las estrategias de diagnóstico y de tratamiento.

2.7 Evaluar las prácticas de prescripción y dispensación y recurrir a grupos de pares o a comparaciones con referencias externas para proporcionar retroalimentación y respaldar prácticas adecuadas de prescripción de antimicrobianos.

2.8 Fomentar la formulación y aplicación de directrices y de algoritmos terapéuticos para promover un uso adecuado de los antimicrobianos.

2.9 Investir a los responsables de los formularios de medicamentos de autoridad para limitar la prescripción a un número adecuado de antimicrobianos seleccionados.

\section{Reglamentación}

2.10 Vincular los requisitos de registro profesional de las personas que prescriben o dispensan antimicrobianos a requisitos de formación continua. uno de ellos es muy variable, dependiendo de la región geográfica, de las circunstancias sociales y del tipo de sistema de salud predominante. Entre los más importantes se encuentran la falta de conocimientos, formación y acceso a la información, la falta de medios para establecer el diagnóstico, el temor a la obtención de malos resultados clínicos, la percepción de las demandas y preferencias de los pacientes, los incentivos económicos a la prescripción, la presión de los colegas y de las normas so- 


\section{Hospitales}

\section{Gestión}

3.1 Establecer programas de control de las infecciones nosocomiales basados en las prácticas óptimas disponibles y velar por que todos los hospitales puedan acceder a ellos.

3.2 Crear comités terapéuticos en los hospitales que puedan supervisar el uso de antimicrobianos en esas instituciones.

3.3 Formular y actualizar periódicamente directrices para el tratamiento y la profilaxis con antimicrobianos, así como formularios hospitalarios de antimicrobianos.

3.4 Monitorizar el uso de antimicrobianos, incluidas las cantidades y modalidades de uso, y poner los resultados a disposición de las personas que prescriben dichos fármacos.

\section{Laboratorios de diagnóstico}

3.5 Garantizar la disponibilidad de servicios de laboratorio microbiológicos que se ajusten al tipo de hospital.
3.6 Garantizar la eficacia y la calidad de las pruebas de diagnóstico, de identificación de microorganismos y de sensibilidad de los principales patógenos, así como la comunicación oportuna de los resultados.

3.7 Garantizar el registro de los datos de laboratorio, preferiblemente en una base de datos, su empleo en informes sobre la vigilancia de la resistencia que resulten útiles desde un punto de vista tanto clínico como epidemiológico, y la comunicación de los resultados a las personas que prescriben y al programa de control de infecciones nosocomiales.

\section{Interacciones con la industria farmacéutica}

3.8 Controlar y vigilar las actividades de promoción de las empresas farmacéuticas en el medio hospitalario, y velar porque esas actividades proporcionen beneficios educativos. ciales, diversos factores relacionados con el ambiente de trabajo, la falta de legislación adecuada o de medios para hacerla cumplir y las deficiencias de la infraestructura de suministro de medicamentos.

\section{HOSPITALES}

Los hospitales son un componente crítico del problema de la resistencia a los antimicrobianos. La combinación de pacientes muy susceptibles con el uso intensivo y prolongado de estos fármacos y las infecciones cruzadas han dado lugar a la aparición de infecciones nosocomiales por microorganismos muy resistentes (bacilos Gram-negativos multirresistentes, enterococos resistentes a la vancomicina, Staphylococcus aureus resistentes a la meticilina y hongos resistentes), algunos de los cuales se han propagado después fuera del medio hospitalario. En los hospitales, es fundamental desarrollar sistemas integrados para mejorar el uso de antimicrobianos, reducir la incidencia y propagación de infecciones nosocomiales y enlazar las decisiones de índole terapéutica con las de suministro farmacéutico. Para ello será preciso capacitar a personas clave y asignar recursos a la vigilancia eficaz, a la lucha contra las infecciones y al apoyo terapéutico.

El elemento clave para reducir la transmisión horizontal de las infecciones en los hospitales consiste en la instauración de buenas prácticas de control de las infecciones, cuyos componentes más importantes son:

- la puesta en práctica de medidas de precaución, como el lavado de las manos y el uso de guantes y batas, y de procedimientos de aislamiento;

- la esterilización y desinfección de los suministros y equipos;

- el uso de técnicas asépticas en los procedimientos médicos y de enfermería;

- la formación del personal sanitario en relación con las técnicas asépticas y los procedimientos de control de las infecciones;

- el mantenimiento de la desinfección y control sanitario del ambiente hospitalario, incluido el aire;

- la vigilancia activa de las infecciones y de la resistencia a los antimicrobianos, el análisis de los datos y la comunicación de la información obtenida al personal sanitario; 


\section{Administración de antimacrobianos a los animales destinados al consumo humano}

\section{Resumen}

4.1 Exigir la obligatoriedad de prescripción de todos los antimicrobianos que se utilizan en la lucha contra las enfermedades de los animales destinados al consumo humano.

4.2 Si no se dispone de una evaluación de su seguridad para la salud pública, interrumpir o reducir paulatinamente el uso en la estimulación del crecimiento animal de los antimicrobianos que también se utilicen para el tratamiento de seres humanos.

4.3 Crear sistemas nacionales de vigilancia de la administración de antimicrobianos a los animales destinados al consumo humano.
4.4 Realizar evaluaciones de la seguridad de los antimicrobianos antes de otorgar las licencias, tomando en consideración la potencial resistencia a los medicamentos destinados a los humanos.

4.5 Monitorizar la resistencia para detectar nuevos problemas sanitarios y tomar medidas correctivas para proteger la salud humana.

4.6 Formular directrices para los veterinarios con el fin de reducir la administración excesiva o indebida de antimicrobianos a los animales destinados al consumo humano.
- la identificación e investigación de los brotes de infección.

Lamentablemente, la prescripción de antimicrobianos es a menudo irracional, incluso en los hospitales. Para muchos médicos, la principal fuente de información es la literatura proporcionada por los representantes de la industria farmacéutica, que tiene menos probabilidades de ser objetiva que las directrices terapéuticas nacionales o regionales.

La existencia en los hospitales de un comité de terapéutica se considera un elemento clave para el control del uso de antimicrobianos en este ámbito. Entre sus actividades destacan:

- la elaboración por escrito de políticas y directrices sobre el uso correcto de los antimicrobianos, basadas en los datos locales de la vigilancia de la resistencia;

- la selección y provisión a la farmacia del hospital de los antimicrobianos apropiados para las necesidades locales;

- el establecimiento de lazos formales con el comité de control de infecciones;

- la definición de un programa de supervisión del uso de antimicrobianos;

- la supervisión del uso de antimicrobianos mediante un sistema de vigilancia de las cantidades utilizadas y de las indicaciones de uso.
Los formularios hospitalarios (listas de fármacos habitualmente almacenados en la farmacia del hospital) constituyen una forma de reducir el uso indebido de antimicrobianos y de contener los gastos. Junto con las directrices clínicas, estos formularios estimulan el uso apropiado de los fármacos preferidos. Sin embargo, todavía no se ha determinado su eficacia en lo que se refiere a la reducción de la aparición de resistencia.

Los laboratorios de microbiología de los hospitales tienen un papel muy importante en la detección y vigilancia de la resistencia a los antimicrobianos. Deben proporcionar pruebas diagnósticas de calidad para identificar correctamente las infecciones y la susceptibilidad a los antimicrobianos, con el fin de guiar la selección de los tratamientos más apropiados. El retraso o error de los resultados diagnósticos conduce frecuentemente a la prolongación del tratamiento empírico.

\section{ADMINISTRACIÓN DE ANTIMICROBIANOS A LOS ANIMALES DESTINADOS AL CONSUMO HUMANO}

Cada vez son más numerosas las pruebas científicas que relacionan la administración de antimicrobianos a los animales destinados al consumo humano con la resistencia de los agentes patógenos comunes. La resistencia tiene consecuencias para la 


\section{Gobiernos nacionales y sistemas de salud}

\section{Actividades de sensibilización y acción} intersectorial

5.1 Declarar prioridad nacional la contención de la resistencia a los antimicrobianos.

- Crear un grupo de trabajo intersectorial nacional, integrado por profesionales de la salud, veterinarios, agrónomos, fabricantes de productos farmacéuticos, miembros del gobierno, representantes de los medios de comunicación, consumidores y otras partes interesadas) para sensibilizar a la opinión pública acerca de la resistencia a los antimicrobianos, organizar la recogida de datos y supervisar a los grupos de trabajo locales. Por razones prácticas, es posible que estos grupos de trabajo tengan que ser gubernamentales y recibir información de numerosos sectores.

- Asignar recursos para promover la realización de las intervenciones de contención de la resistencia, que deben incluir el uso adecuado de los antimicrobianos, la lucha contra las infecciones y su prevención, y las actividades de investigación.

- Crear indicadores para monitorear y evaluar los efectos de la estrategia de contención de la resistencia a los antimicrobianos.

\section{Reglamentación}

5.2 Establecer un sistema de registro eficaz de los puntos de dispensación.

5.3 Convertir los antimicrobianos en medicamentos para los que se exige receta médica, salvo en circunstancias especiales en las que podrían dispensarse por recomendación de un profesional sanitario cualificado.

5.4 Relacionar la condición de medicamento de venta con receta médica con los reglamentos relativos a la venta, suministro, dispensación y actividades de promoción de los antimicrobianos; establecer mecanismos para facilitar a los médicos el cumplimiento de las disposiciones y sistemas para vigilarlo.

5.5 Velar por que únicamente se autorice la venta de los antimicrobianos que cumplan con las normas internacionales de calidad, seguridad y eficacia.
5.6 Declarar obligatorio que los fabricantes reúnan datos sobre la distribución de antimicrobianos (incluidas la importación y exportación) e informen al respecto.

5.7 Crear incentivos económicos para un uso adecuado de los antimicrobianos.

\section{Políticas y directrices}

5.8 Elaborar y actualizar periódicamente directrices terapéuticas nacionales y fomentar su aplicación.

5.9 Establecer una lista de medicamentos esenciales que se ajuste a las directrices terapéuticas nacionales y garantizar la accesibilidad y la calidad de los medicamentos.

5.10 Aumentar la cobertura de las vacunaciones y potenciar otras medidas de prevención de las enfermedades, de modo que disminuya la necesidad de usar antimicrobianos.

\section{Educación}

5.11 Aprovechar al máximo y mantener la eficacia de la lista de medicamentos esenciales y de las directrices terapéuticas mediante programas educativos para profesionales de la salud y estudiantes de medicina y de posgrado. El contenido de los programas deberá incluir la importancia de la contención de la resistencia y de usar debidamente los antimicrobianos.

5.12 Garantizar que las personas que prescriben antimicrobianos tengan acceso a la documentación autorizada sobre la prescripción de los fármacos específicos.

\section{Vigilancia de la resistencia,}

uso de los antimicrobianos y morbilidad

5.13 Designar o crear laboratorios de microbiología de referencia para coordinar una vigilancia eficaz y epidemiológicamente acertada de la resistencia de los patógenos comunes, tanto en la comunidad como en los hospitales y otros servicios de atención sanitaria. Estos laboratorios deberían cumplir, como mínimo, los requisitos de la recomendación 3.6.

5.14 Adaptar y aplicar los modelos de la OMS para la vigilancia de la resistencia a los antimicrobianos y velar por que se envíen los datos al grupo de trabajo especial nacional e intersec- 


\section{Gobiernos nacionales y sistemas de salud (continuación)}

torial, a las autoridades encargadas de las directrices terapéuticas nacionales y de las políticas de medicamentos y a quienes prescriben los fármacos.

5.15 Establecer sistemas para la vigilancia del uso de antimicrobianos en los hospitales y en la comunidad, y relacionar los resultados con los datos sobre la resistencia y la vigilancia de las enfermedades.

5.16 Establecer sistemas de vigilancia de los principales síndromes y enfermedades infecciosas, ajustándose a las prioridades del país, y vincular esta información con otros datos sobre la vigilancia. salud de los animales, y también para la salud de los seres humanos cuando los patógenos se introducen en la cadena alimentaria. Los factores que inciden en este uso de los antimicrobianos, ya sea por motivos terapéuticos, profilácticos o de estimulación del crecimiento, son complejos y las intervenciones necesarias deberán realizarse de forma coordinada. Los principios rectores del uso apropiado de los antimicrobianos y de la contención de la resistencia en este ámbito son semejantes a los que se aplican a los seres humanos. En una reunión consultiva de la OMS celebrada en Ginebra en junio de 2000 se adoptaron los principios de la OMS para la contención de la resistencia a los antimicrobianos en los animales destinados al consumo humano ${ }^{2}$, que constituyen un marco de recomendaciones para reducir la administración excesiva e indebida de antimicrobianos a estos animales, con el objetivo de proteger la salud humana. En ese documento figura una descripción completa de todas las recomendaciones y aquí únicamente se recoge un resumen de las mismas. Los antimicrobianos también son de uso corriente en otros campos, como la horticultura y la acuicultura, pero sus riesgos para la salud humana son menos conocidos y no se han incluido en este documento.

El uso de antimicrobianos en los animales destinados al consumo humano puede afectar a la salud humana debido a la presencia de residuos de los fármacos en los alimentos y, sobre todo, a la selección de microorganismos resistentes en los ani-

World Health Organization. WHO global principles for the containment of antimicrobial resistance in animals intended for food. 2000. WHO/CDS/CSR/APH/2000.4. Disponible en: http://www.who. int/emc/diseases/zoo/who_global_principles.html males. Las consecuencias de dicha selección incluyen el aumento del riesgo de que los patógenos resistentes sean transferidos al ser humano por el contacto directo con los animales o por el consumo de alimentos o aguas contaminadas y la transferencia de genes de resistencia de la flora bacteriana animal a la humana.

Los factores implicados en la aparición de resistencia a los antimicrobianos en los animales destinados al consumo humano parecen ser los mismos que en los humanos, pero hay algunas peculiaridades importantes. En primer lugar, de los tres usos de los antimicrobianos en los animales (estimulación del crecimiento, profilaxis y tratamiento), los de mayor peso son los dos primeros. Además, debido a la inexistencia de servicios de diagnóstico microbiológico, el uso terapéutico es generalmente empírico, y la identificación de algún animal enfermo suele llevar al tratamiento de toda la población, al contrario de lo que ocurre en los humanos, en los que el tratamiento es individualizado. Otro factor muy importante consiste en el hecho de que, en algunos países, hasta un $40 \%$ de los ingresos de los veterinarios proceden de la venta de fármacos.

\section{GOBIERNOS NACIONALES Y SISTEMAS DE SALUD}

Las políticas de salud gubernamentales y los sistemas de salud en los que se aplican son fundamentales para determinar la eficacia de las intervenciones dirigidas a contener la resistencia a los antimicrobianos. También son requisitos indispensables la voluntad nacional de entender y atajar el problema y de designar autoridades y competen- 


\section{Desarrollo de medicamentos y vacunas}

6.1 Fomentar la cooperación entre la industria farmacéutica, los organismos gubernamentales y las instituciones académicas para la investigación de nuevos medicamentos y vacunas.

6.2 Fomentar los programas de desarrollo de medicamentos que traten de optimizar los regímenes terapéuticos en lo que se refiere a su seguridad, eficacia y riesgo de selección de organismos resistentes.

6.3 Establecer incentivos para que la industria invierta en la investigación y el desarrollo de nuevos antimicrobianos.

6.4 Estudiar el establecimiento o la aplicación de procedimientos acelerados de autorización de la comercialización de nuevos agentes seguros.
6.5 Estudiar la aplicación de un régimen de medicamentos huérfanos cuando se disponga de uno y sea pertinente.

6.6 Permitir la exclusividad, durante un tiempo limitado, de las nuevas fórmulas o indicaciones para el uso de antimicrobianos.

6.7 Armonizar los derechos de propiedad intelectual para que se cuente con una protección adecuada de las patentes de nuevos agentes antimicrobianos y vacunas.

6.8 Buscar fórmulas de asociación innovadoras con la industria farmacéutica para mejorar el acceso a los medicamentos esenciales más recientes. cias. Para que una acción sea eficaz, es necesario elaborar y aplicar una reglamentación adecuada y asignar los recursos oportunos para la educación y la vigilancia. La interacción constructiva con la industria farmacéutica es fundamental, tanto para la concesión de licencias y la promoción y la comercialización de los antimicrobianos existentes, como para alentar la elaboración de nuevos medicamentos y vacunas. Para una mayor claridad, las intervenciones relativas a la interacción con la industria farmacéutica se exponen en recomendaciones separadas (puntos 6 y 7).

Los gobiernos y los sistemas de salud pueden desempeñar un papel importante a la hora de reducir la aparición y el desarrollo de la resistencia a los antimicrobianos mediante la elaboración de políticas y leyes sobre el desarrollo, aprobación, distribución y venta de estos fármacos. Los aspectos reglamentarios clave incluyen la habilitación de los profesionales, la capacidad para prescribir y dispensar medicamentos, su registro y aprobación, la calidad de los productos, su precio y los sistemas de suministro de medicamentos.

La estructura y organización de los sistemas de salud pueden ser factores que determinan la fiabilidad y viabilidad de los sistemas de recogida de datos acerca del uso de antimicrobianos, de la vigilancia de la resistencia y del impacto clínico de esta.
Además, pueden tener una influencia directa en los currículos de los estudios de medicina, en la existencia y mantenimiento de sistemas de registro de todos los profesionales sanitarios y en la atención prestada a su formación continuada.

La OMS elaboró en 1977 la primera lista de medicamentos esenciales, con el objetivo de promover la disponibilidad de varios fármacos seleccionados y su uso racional. Desde entonces, esta lista ha sido revisada periódicamente y le ha servido a los países como guía para establecer sus políticas nacionales de medicamentos. En la actualidad hay más de 120 países que han elaborado sus propias listas de medicamentos esenciales, que parecen haber mejorado el acceso a estos fármacos.

\section{DESARROLLO DE MEDICAMENTOS Y VACUNAS}

La industria farmacéutica es la principal fuente de nuevos antimicrobianos y de nuevas modalidades de prevención de las enfermedades infecciosas. Por consiguiente, es fundamental que haya incentivos para que las compañías farmacéuticas inviertan en la investigación y desarrollo en estos campos, aunque existan otros tipos de medicamentos más rentables. Esto es particularmente impor- 


\section{Promoción de los medicamentos}

7.1 Hacer obligatorio que la industria farmacéutica se atenga a los códigos de prácticas nacionales o internacionales para las actividades de promoción.

7.2 Garantizar que los códigos de prácticas nacionales o internacionales abarquen la publicidad dirigida al consumidor, incluida la publicidad por Internet.
7.3 Establecer sistemas para la vigilancia del cumplimiento de la legislación sobre actividades de promoción.

7.4 Identificar y eliminar los incentivos económicos que fomentan el uso inadecuado de los antimicrobianos.

7.5 Concienciar a las personas que prescriben antimicrobianos de que la promoción conforme a la hoja de datos no constituye, necesariamente, un uso adecuado de esos fármacos. tante con respecto a las vacunas y a los antimicrobianos que se utilizarán sobre todo en países con escasos recursos. La industria farmacéutica es bien consciente del problema de la resistencia a los antimicrobianos, que también le afecta de forma negativa, pero al mismo tiempo puede preocuparle el hecho de que el uso apropiado de estos fármacos reduzca las ventas. En la actualidad se están analizando diversos tipos de incentivos a las compañías farmacéuticas. Una forma de estimular el descubrimiento de nuevos fármacos consiste en la cooperación entre la industria y las instituciones académicas. La cooperación entre organismos públicos y empresas privadas es otro mecanismo que puede acelerar el descubrimiento de nuevos fármacos para necesidades no cubiertas y especialmente para aquellas con menores perspectivas comerciales.

Las vacunas tienen una enorme importancia en la contención de la resistencia a los antimicrobianos, dado que permiten reducir la incidencia de las enfermedades infecciosas y de los estados de portador de cepas resistentes. Otros aspectos importantes en relación con la industria farmacéutica son los mecanismos de aprobación rápida de nuevos fármacos, los acuerdos sobre medicamentos huérfanos y los mecanismos de protección de los derechos de propiedad intelectual.

\section{PROMOCIÓN DE LOS MEDICAMENTOS}

El control gubernamental de las actividades de promoción de los medicamentos y del cumplimiento de la legislación y de los códigos deontoló- gicos son factores muy importantes para lograr el uso apropiado de los antimicrobianos. Las estrechas relaciones entre la promoción de los medicamentos, los hábitos de prescripción y las ventas han quedado desmostradas en varios estudios. Dado que la promoción incrementa el uso, es de suponer que influye sobre la prevalencia de la resistencia, sobre todo si da lugar a un aumento del uso indebido.

La publicidad y la literatura distribuida por las compañías farmacéuticas son fuentes de información con gran influencia entre los profesionales sanitarios. En muchos casos constituyen la única fuente de información de fácil acceso. En ausencia de legislación y de medidas para garantizar su cumplimiento, la industria farmacéutica podría presentar una información muy selectiva y sesgada sobre la eficacia y seguridad de los medicamentos.

Por otra parte, es bien sabido que los profesionales sanitarios se sienten a menudo presionados por los pacientes para que prescriban antimicrobianos ante infecciones menores que en realidad no necesitan tratamientos específicos. En países donde los antimicrobianos solo se pueden dispensar con receta médica, la publicidad dirigida directamente al público puede incrementar estas presiones sobre los profesionales. Un problema más reciente es el de la publicidad en Internet, que está penetrando cada vez más en el mercado y resulta muy difícil de controlar debido a la naturaleza del medio.

Las actividades de promoción que se consideran aceptables y las reglamentaciones al respecto varían según los países. 


\section{Aspectos internacionales de la contención de la resistencia a los an- timicrobianos}

8.1 Fomentar la colaboración entre los gobiernos, las organizaciones no gubernamentales, las asociaciones profesionales y los organismos internacionales para entender la importancia de la resistencia a los antimicrobianos, lanzar mensajes congruentes, sencillos y exactos sobre la importancia del uso de los antimicrobianos, la resistencia a los antimicrobianos y su contención, y aplicar estrategias para contener la resistencia.

8.2 Considerar de utilidad pública mundial para la salud la información obtenida mediante la vigilancia del uso y de la resistencia a los antimicrobianos, así como su contención, a la que deben contribuir todos los gobiernos.

8.3 Alentar a los gobiernos, a las organizaciones no gubernamentales, a las asociaciones profesionales y a los organismos internacionales a apoyar la creación de redes que cuenten con personal capacitado e infraestructuras adecuadas y que puedan asumir una vigilancia epidemiológica válida de la resistencia y del uso de antimicrobianos, a fin de disponer de información para la contención óptima de la resistencia.

8.4 Propugnar la donación de medicamentos de conformidad con las directrices de las Naciones Unidas.
8.5 Fomentar la creación de equipos de inspección internacionales cualificados para efectuar evaluaciones válidas de las fábricas de productos farmacéuticos.

8.6 Fomentar una estrategia internacional para luchar contra la falsificación de antimicrobianos que se ajuste a las directrices de la OMS. ${ }^{3}$

8.7 Fomentar estrategias innovadoras con respecto a los incentivos para el desarrollo de nuevos productos farmacéuticos y vacunas para las enfermedades desatendidas.

8.8 Crear una base de datos internacional de organismos de financiación de investigaciones que estén interesados en la resistencia a los antimicrobianos.

8.9 Crear nuevos programas y consolidar los existentes, para que los investigadores mejoren los proyectos, la preparación y la ejecución de las investigaciones sobre la contención de la resistencia a los antimicrobianos.

3 World Health Organization. Counterfeit drugs. Guidelines for the development of measures to combat counterfeit drugs. Geneva, World Health Organization, 1999 (WHO/EDM/QSM/ 99.1).

\section{ASPECTOS INTERNACIONALES DE LA CONTENCIÓN DE LA RESISTENCIA A LOS ANTIMICROBIANOS}

Existen múltiples factores de carácter mundial que influyen en la epidemiología de las enfermedades infecciosas, como la urbanización creciente, la contaminación y los cambios ambientales, los cambios demográficos o la epidemia de sida. El aumento de la incidencia de las infecciones conduce al aumento del uso de los antimicrobianos y, por consiguiente, a una mayor presión selectiva en favor de los microorganismos resistentes. A su vez, la expansión del comercio mundial y de los viajes ha acelerado la propagación intercontinental de las enfermedades infecciosas y de los microorganismos resistentes. Todo esto requiere acciones internacionales concertadas para contener la resistencia y una mayor cooperación entre las organizaciones internacionales y los gobiernos y organismos gubernamentales, las sociedades profesionales y las organizaciones no gubernamentales nacionales. Para incrementar las posibilidades de éxito de las estrategias nacionales se le debería conceder una alta prioridad al intercambio de información entre los países. La colaboración internacional en el campo de la vigilancia de la resistencia también podría ser valiosa, pero todavía no existen mecanismos formales ni instrumentos legales internacionales para llevarla a cabo.

Las donaciones de medicamentos por parte de las compañías farmacéuticas han tenido efectos 
muy importantes en la disponibilidad de tratamientos en situaciones de emergencia complejas y en los programas de eliminación y erradicación de algunas enfermedades (lepra, oncocercosis, filariasis linfática) en países con escasos recursos. Aunque hay que alentar estas donaciones, en algunas situaciones puede ser necesario coordinarlas mejor y mejorar la selección de los medicamentos proporcionados, así como su distribución y accesibilidad, con el fin de evitar la duplicación y el despilfarro. Se deberían adoptar medidas internacionales para garantizar que todas las donaciones cumplan las normas existentes. ${ }^{4}$

Los antimicrobianos se encuentran entre los fármacos falsificados con mayor frecuencia y las autoridades nacionales e internacionales deberían colaborar para garantizar el cumplimiento de las leyes al respecto. Otros aspectos considerados en este documento son las inspecciones internacionales de la fabricación de medicamentos, los códigos internacionales de buenas prácticas de mercadotecnia, la cooperación internacional en la investigación y desarrollo de nuevos fármacos y vacunas o el apoyo internacional a la contención de la resistencia a los antimicrobianos en el ámbito nacional.

El documento concluye con la exposición de un modelo para la puesta en práctica de esta estrategia mundial de la OMS, en el que, mediante un método escalonado, se trata de destacar las intervenciones más importantes y de identificar una secuencia lógica para su puesta en práctica. Sin em-

4 World Health Organization. Interagency guidelines. Guidelines for drug donation, revised 1999. Geneva: WHO; 1999 (WHO/EDM/ PAR/99.4). Resumido en Pan Am J Public Health 2000;7:131-134. bargo, ese modelo no pretende más que servir como guía, una vez que las decisiones adoptadas por cada gobierno deberán tener en cuenta las circunstancias, los sistemas de salud y las enfermedades más frecuentes en cada país.

\section{SYNOPSIS}

\section{World Health Organization strategy to contain resistance to antimicrobial drugs}

This piece summarizes a report from the World Health Organization on the WHO Global Strategy for Containment of Antimicrobial Resistance. The Strategy provides a framework for interventions aimed at minimizing the appearance and spread of resistant microorganisms, through a series of measures designed to: reduce morbidity and the spread of infections, improve access to appropriate antimicrobial drugs, improve the use of antimicrobial drugs, strengthen health systems and their surveillance capacity, enforce regulations and legislation, and promote the development of new drugs and vaccines. The Strategy report makes a series of recommendations for interventions related to: patients and the community in general, those who prescribe and dispense antimicrobial drugs, hospitals, the administration of antimicrobial drugs to animals destined for human consumption, national governments and health systems, the development of drugs and vaccines, the promotion and advertising of drugs, and international aspects of containing resistance to antimicrobial drugs. In addition, the report puts forth a model for implementing the WHO Strategy, in which a step-by-step approach is used to discern the most important interventions and to identify a logical sequence for putting them into place. 